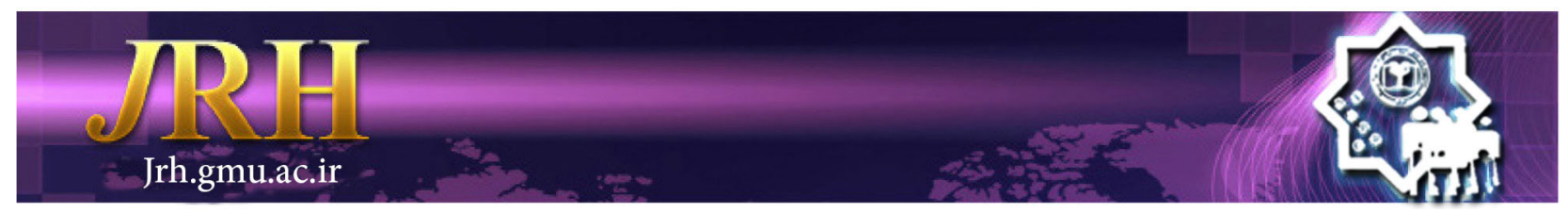

\title{
The impact of information technology on health
}

Alireza Atarodi ${ }^{1}$, Ahmadreza Atarodi ${ }^{2}$

\section{Journal of Research \& Health \\ Social Development \& Health Promotion Research Center \\ Vol. 9, No.3, May \& Jun 2019 \\ Pages: 193- 194 \\ DOI: 10.29252/jrh.9.3.193 \\ Letter to Editor}

1. Department of Knowledge and Information Science, Paramedical College and Social Development \& Health Promotion Research Center, Gonabad University of Medical Sciences, Gonabad, Iran

2. Correspondence to: Student Research Committee, School of Medicine, Gonabad University of Medical Sciences, Gonabad, Iran Email: a.atarodi.b@gmail.com

Received: 3 Feb 2019

Accepted: 9 Mar 2019

How to cite this article: Atarodi A, Atarodi A. The impact of information technology on health. J Research \& Health2019; 9(3): 193194.

\section{Dear Chief in Editor}

Information Technology (IT) is the study of systems especially computers for storing, retrieving, and sending information. It uses any networking and other physical devices, infrastructures to secure and exchange all forms of electronic data. IT is used globally as a major portion of daily life and we use it nearly every day within organizations for many reasons. Our computers, mobile phones and most of other devices we use are IT forms. We depend on them everywhere and every day to do our works more efficiently. We use IT for big data, cloud computing, internet, enterprise software, artificial intelligence, blockchain, cyber security, virtual assistance, augmented reality, 3-D printing and more. Now, it seems that a profound revolution has taken place in communication technology today which has influenced the pace and way of relationship in the world. IT has affected all areas and changed the environment so that it was not available for anybody before and also has created the opportunity for human to exchange the information and knowledge easily and in a piece of time [1]. Another aspect of information technology is its contribution to health and health issues. Health information technology (HIT) is a basic factor in care delivery and health care related affairs. In general, HIT refers to electronic information systems, which is used to create, store, transmit, receive, share and analyze health data and information through Electronic Health Records (EHRs), Health Information Exchange (HIE), and telemedicine or telehealth in hospitals, clinics and private sectors. Some of HIT applications are as the following.
Some examples of different applications of HIT. Storage, management, share, analysis and transmission of health data and information: including electronic and personal health record systems that contain patient-specific data that can be accessed by patients and professionals (data sharing) for clinical, administrative and/or personal (patient-specific) purposes. The analysis of large population data sets aimed at improving population health through EHRs allow doctors to keep track of their patients' health information (Personal Health Records (PHRs) everywhere and every time needed) better.

Decision support: For clinicians, this might include electronic decision support systems that provide diagnostic or treatment advice to facilitate medication prescriptions. For patients, this might include providing personal decision support through the use of, for example, Internet and mobile apps to monitor clinical conditions and inform decision making for instance in chronic disease management and also automated clinical alerting and Electronic prescribing (E-prescribing).

Facilitating Long-distance care: Telemedicine, which involves communication between healthcare professionals and patients (e.g. remote consultations through telecare or teletherapy applications such as monitoring vital signs in patients' homes by telephones or 
internet [2].

Privacy and security. All of these electronic systems can increase the protections of patients' health information. For example, electronic information may be encrypted so that only authorized people can read it. HIT can also make it easier to record and track who has accessed the information. A systematic review on over $60 \%$ of studies have reported that HIT was associated with improvement in one or most aspects of care, especially in the effectiveness and efficiency of health care [3].

Information and knowledge are the most fundamental factor in the growth and development of a community health. Some studies in Europe countries and in Ardabil, Zabol and Kashan universities claimed that using HIT in health sector is highly effective and the society attitude toward its implementation and development is mostly positive [4].

Health information technology presents numerous opportunities for improving and transforming healthcare which includes; reducing human errors (which is the cause of death for approximately 12,000 patients every year) [2], improving clinical outcomes, facilitating care coordination, improving practice efficiencies, and tracking data over time. Since the original Institute of Medicine (IOM) report was published, there has been an accelerated development and adoption of health information technology with different degrees of evidence about the impact of health information technology on patient safety (which is preventing medical errors and increasing health care accuracy and procedural correctness) [2-5]. Educational evaluation, especially medical sciences, is sensitive and important that helps to determine the strengths and weaknesses of the HIT. Based on the results by developing positive aspects and removing deficiencies, appropriate steps can be taken to improve HIT for more success in all issues. However the use of technology can also have negative effects on individuals' health such as their vision problems, hearing loss, feeling tired and neck strain. Besides, implementation of HIT brings time and financial concerns as well as physical disabilities, insufficient computer skills, concerns about confidentiality and lack of awareness of HIT potential benefits. However, there are some steps that can be taken to help deal with these challenges as well.

Understanding HIT ability and capacity for training programs is an attempt to create a new educational opportunity for health centers, since HIT has had a greater impact on the various aspects of human life from the past. Providers and users' viewpoints are an essential information element for developing a creative HIT training program, new idea and turning it into an appropriate educational opportunity for developing HIT. Health in all aspects and sectors is based on change of individuals' attitudes toward the issue of having sufficient information and knowledge about HIT benefits. The widespread use of HIT can help improve the quality of patient care, reduce medical preventable errors and make more cost savings through greater efficiency based on understanding its benefits. Therefore, it seems that we should confirm the impact of HIT on health and then do our best to enjoy its advantages for human health.

\section{References}

1- Tavakolizadeh J, Atarodi A, Ahmadpour S, Pourgheisar A. The prevalence of excessive mobile phone use and its relation with mental health status and demographic factors among the students of Gonabad University of medical sciences in 2011-2012. Razavi International Journal of Medicine2014; 2(1): e15527.

2- Black A, Car J, Pagliari C et al. The impact of health on the quality and safety of health care: a systematic overview. PLoS Med2011; 8: 71238

3- Sood SH, McNeil K. How is health information technology changing the way we deliver NHS hospital care? Future Healthcare Journal2017; 4(2): 117-20.

4- Haghiri E, Hesam S, Ataiorcid J, Jadidi R, Etebar I. Effects of information technology on the improvement of health systems from the viewpoint of executive managers. $J$ Paramedical and Rehabilitation of Mashhad2017; 7(1): 47-56.

5- Alotaibi YK, Federico F. The impact of health information technology on patient safety. Saudi Med $J 2017$; 38(12): 1173-80.

Copyright $(2016$ ASP Ins. This open-access article is published under the terms of the Creative Commons Attribution-NonCommercial 4.0 International License which permits Share (copy and redistribute the material in any medium or format) and Adapt (remix, transform, and build upon the material) under the Attribution-NonCommercial terms. 